drug for an uncommon disease market protection from identical competitors for seven years. Few question that Avonex and Betaseron are structurally similar, but is Avonex dissimilar enough to escape the Orphan Drug Act? One consideration is that some Betaseron users develop antibodies that neutralize its biological effects, whereas antibodies appear to be somewhat less of a problem with Avonex.

Neither drug is a cure for MS, and neither drug eliminates the attacks associated with the relapsing form of the disease. In the United States, 250,000-300,000 people have
MS, and about half have relapsing (rather than chronic progressive) MS. The mechanism of action of $\beta$-IFN is not understood, but both drugs probably act in a similar manner. But are there sufficient differences in clinical effectiveness and side effect profiles to warrant approval of a competing product?

A comparison of the trial data shows that, overall, Avonex slowed the progression of disease more than Betaseron, although individual patients might not notice a marked benefit of one drug over the other. Both drugs reduce relapses, or sporadic

\title{
Approved or advanced experimental MS therapies
}

Several companies are currently jostling for a share of the lucrative therapeutic market for multiple sclerosis (MS), which affects an estimated 1.1 million people worldwide. The interferons (IFN) currently in the clinic are IFN- $\beta$ la (produced in mammalian cells) or IFN- $\beta 1 \mathrm{~b}$ (produceed in bacterial cells). The increased efficacy and injection-site tolerance of IFN- $\beta$ la compared to IFN- $\beta$ lb was cited as justification for the FDA's recent approval of Avonex, despite Berlex's (Wayne, NJ) orphan drug exclusivity for Betaseron. Ares Serono (Geneva, Switzerland) also has two IFN- $\beta$ products, Rebif and Frone, both currently in phase III trials.

Teva (Petach Tikva, Israel) is currently awaiting approval from the US and UK regulatory authorities for Copaxone, a compound that resembles myelin, and inhibits myelin destruction in MS. Two neurotrophic factors (Bio/Technology 13:1167-1171, 1995), brain-derived neurotrophic factor (BDNF) from Amgen (Thousand Oaks, CA), and insulin-like growth factor I (IGF-I) from Genentech (South San Francisco, CA), which have activity in other neurodegenerative indications, are also progressing through trials

\begin{tabular}{|c|c|c|c|}
\hline \multicolumn{4}{|c|}{ MS therapies either in or approaching the clinic. } \\
\hline R8D company & Type of therapeutic & Product & Status \\
\hline Berlex & IFN- $\beta 1 b$ & Betaseron & In the clinic \\
\hline Biogen & IFN- $\beta$ 1a & Avonex & In the clinic \\
\hline \multirow[t]{2}{*}{ Ares-Serono } & IFN- $\beta 1 \mathrm{a}$ & Rebif & Phase III \\
\hline & IFN- $\beta 1 \mathrm{~b}$ & Frone & Phase III \\
\hline Teva & Myelin mimic & Copaxone & Phase III completed \\
\hline British Biotech & Platelet-activating factor inhibitor & Lexipafant & Phase II \\
\hline Genentech & Neurotrophic factor & IGF-I & Phase III \\
\hline Amgen & Neurotrophic factor & BDNF & Phase I/II \\
\hline GlaxoWellcome & Mab & CAMPATH-1H & Phase $1 / 11$ \\
\hline Athena & Mab & Antegren. & Phase II \\
\hline Centocor & Mab & Centaratm & Phase II \\
\hline IDEC & Mab & IDEC-CE9.1 & Phase II \\
\hline Autolmmune & T-cell blocker & Myloral & Phase II \\
\hline
\end{tabular}

for MS. Several companies have developed monoclonal antibodies (Mabs) to target receptors on autoreactive T cells; many of these Mabs are now in phase I/II trials. Another approach is to immunize with peptides that stimulate regulatory $\mathrm{T}$ cells to bind and downregulate autoreactive $\mathrm{T}$ cells, as is the case for AutoImmune's (Lexington, MA) Myloral. A number of other companies, including British Biotech (Oxford, UK), Biocryst Pharmaceuticals (Birmingham, AL), and Synthelabo (Le Plessis Robinson, France) are developing antiinflammatory small molecules for MS, which can be taken orally. Guilford Pharmaceuticals (Baltimore, MD) has recently announced promising data for its small-molecule neuroimmunophilin ligands, which have been shown to promote neuronal regeneration and remyelination.

Andrew Marshall

exacerbations of the disease, over time. Betaseron appears to have the edge in relapse reduction.

Both drugs cause flu-like side effects but, perhaps significantly, Avonex appears to cause much fewer injection-site reactions. This difference is exacerbated by the different dosing regimens of the two drugs: Betaseron is administered every other day subcutaneously, and Avonex is given once a week via intramuscular injection.

Biogen says the data show that Avonex is the only drug to slow the progression of disability in MS. Berlex argues that identifying clinical differences between the two drugs demands head-to-head comparative clinical studies. None has yet been performed.

"For scientific reasons, it's very difficult to compare the results of two different clinical trials done years apart, using different investigators and different kinds of patients, and totally different experimental methods," says Latts. "That, scientifically, is inappropriate."

He points out that the placebo groups in the two trials do not have the same outcomes, suggesting that patient selection or treatments may have varied simply because of the investigators involved. Nevertheless, the FDA has compared the two sets of data in making its approval of Avonex. Latts says it is a matter for debate "whether [the FDA] had the authority to do that. .."

Kathryn Bloom, Biogen's director of corporate communication, put aside questions of the approvability of the company's drug. "They are different drugs with different dose regimens," she said.

The FDA will not comment on the pending lawsuit. In its approval statement, it has cited the clinical findings that patients receiving Biogen's Avonex are $37 \%$ less likely to accumulate signs of physical disability compared to the placebo group, symptom flare-ups occur less often in the treatment group, and magnetic resonance imaging reveals fewer brain lesions. "This new product has been allowed to enter the market because it differs from IFN $\beta-1 b$ based on an absence of reports of injection-site necrosis," the statement reads. These reactions have been blamed for Betaseron's inability to capture a larger share of the MS market and for patients stopping treatment.

"We believe that we have orphan drug protection for Betaseron and that the Avonex product is covered by that orphan drug protection," says Latts. "We are pursuing our rights in this matter via litigation, and I think we will soon get a chance to present the arguments in court as to why the FDA erred in approving Avonex."

Vicki Glaser 